\title{
PLANE WAVES IN THERMO-VISCOELASTIC MATERIAL WITH VOIDS UNDER DIFFERENT THEORIES OF THERMOELASTICITY
}

\author{
S.K. TOMAR and N. GOYAL* \\ Department of Mathematics, Panjab University \\ Chandigarh - 160 014, INDIA \\ E-mails: sktomar@pu.ac.in; goyal.n104@gmail.com
}

\author{
A. SZEKERES \\ Department of Applied Mechanics, BME \\ Budapest 1111, HUNGARY \\ E-mail: szekeres@mm.bme.hu
}

\begin{abstract}
Propagation of time harmonic plane waves in an infinite thermo-viscoelastic material with voids has been investigated within the context of different theories of thermoelasticity. The equations of motion developed by Iesan [1] have been extended to incorporate the Lord-Shulman theory (LST) and Green-Lindsay theory (GLT) of thermoelasticity. It has been shown that there exist three coupled dilatational waves and an uncoupled shear wave propagating with distinct speeds. The presence of thermal, viscosity and voids parameters is responsible for the coupling among dilatational waves. All the existing waves are found to be dispersive and attenuated in nature. The phase speeds and attenuation coefficients of propagating waves are computed numerically for a copper material and compared under different theories of thermo-elasticity. The expressions of energies carried along each wave have also been derived. All the computed numerical results have been depicted through graphs. It is found that the influence of CT and GLT is almost same on wave propagation, while LST influences the wave propagation differently.
\end{abstract}

Key words: thermo-visco-elasticity, voids, waves, phase speed, attenuation, energy.

\section{Introduction}

There are three types of theories on thermo-elasticity generally available in the literature, namely, uncoupled, coupled and generalized. The classical uncoupled theory of thermoelasticity is based on two phenomena (shortcomings) which are not compatible with physically observed experiments. According to the first phenomenon, the energy equation of this theory does not contain any elastic term and according to the other phenomenon, the thermal wave propagates with infinite speed (parabolic type). When the elastic solid is subjected to a thermal disturbance, the effect is felt in a location far from the source instantaneously, which is absurd. The first shortcoming was taken care by Biot [2] by introducing the theory of coupled thermoelasticity. The governing equations of this new theory were coupled, but still the theory contained the second shortcoming. The shortcoming corresponding to infinite speed of propagation in the uncoupled theory of thermoelasticity was investigated by several researchers, e.g. Szekeres [3], Ferkas and Szekeres [4] and Chandrasekharaiah [5]. In particular, Szekeres [3] has nicely explained that the Fourier law of heat conduction is the most effective model in physics in spite of that it has fundamental errors. These authors modified the Fourier law of heat conduction, but showed that it leads to a hyperbolic differential equation and the speed of propagation becomes finite. Almost all modifications were in need of at least one relaxation time, whose experimental determination was a challenging task and almost impossible [see Szekeres and

\footnotetext{
* To whom correspondence should be addressed
} 
Szalontay [6]]. Several generalizations of Biot's theory of thermoelasticity have been developed by the researchers (see Hetnarski and Ignaczak [7]). The first generalization was made by Lord and Shulman [8], who obtained a wave like heat equation by incorporating a flux rate term into the classical Fourier's law of heat conduction. In this theory, the constitutive relations contain one relaxation time. The second generalization is given by the Green and Lindsay theory [9] [GLT] by introducing temperature rate among constitutive variables that includes two thermal relaxation times and does not violate the classical law of heat conduction when the body under consideration has a centre of symmetry. Green and Naghdi theory [10] [GNT] proposed the third generalization of the coupled theory of thermoelasticity, which is the theory of thermoelasticity without energy dissipation. In this theory, the classical Fourier law is replaced by heat flux rate-temperature gradient relation. This theory admits uncoupled thermoelastic waves. The fourth generalization was formulated by Hetnarski and Ignaczak (1996) which is known as a low temperature thermoelasticity theory [LTT]. The fifth generalization to thermoelasticity theory was proposed by Tzau [11].

Goodman and Cowin [12] introduced the continuum theory of granular materials, in which the concept of distributed body is introduced. Later, Nunziato and Cowin [13] utilized the idea of distributed bodies and developed a nonlinear theory of elastic materials with voids. The linear theory of elastic material with voids was presented by Cowin and Nunziato [14]. The basic idea lying under the theory of elastic material with voids is that the bulk density of the material is written as a product of two entities: one defining the change in void volume fraction and the other defines the density of the matrix material. This representation of the bulk density of the material could introduce an additional kinematic variable in the theory. Several problems concerning waves and vibrations based on the theories of elastic material with voids have been investigated and appeared in the literature. Some notable results have been established by Puri and Cowin [15], Iesan [16], Chandrasekhariah [17, 18], Marin [19], Birsan [20], Chirit a and Scalia [21], Cicco and Diaco [22], Iesan and Nappa [23], Iesan [24], Tomar [25], Ciarletta et al. [26], Ciarletta et al. [27], Svanadze [28] and Chirit a and Danescu [29]. Iesan [30] extended Cowin and Nunzaito theory to incorporate thermal effect and presented the theory of thermoelastic materials with voids. Dhaliwal and Wang [31] presented a heat-flux dependent theory of thermoelasticity with voids. Ciarletta and Scalia [32] studied uniqueness and reciprocity in linear thermoelasticity of materials with voids. Ciarletta and Scarpetta [33] also gave some results on thermo-elasticity for dielectric materials with voids. Iesan [1] further extended the theory of thermoelastic material with voids to incorporate the time rate of strain tensor and time rate of gradient of void volume fraction and presented the theory of thermo-viscoelastic material with voids. Using his theory, Tomar et al. [34] studied the possibility of time harmonic plane wave propagation in an infinite thermo-viscoelastic material with voids. They found that four basic waves can propagate in the material with distinct speeds consisting of three sets of coupled dilatational waves and a lone shear wave. All the waves are found to be dispersive and attenuating. Sharma and Kumar [35] studied one-dimensional plane wave propagation in generalized thermo-viscoelastic material with voids. Their generalization of thermoelasticity consists of CT and LST. They have also constructed the fundamental solution of the system of differential equations in the theory of thermo-viscoelastic medium with voids in case of steady oscillations in terms of elementary functions. Bucur et al. [36] studied the behaviour of plane waves and Rayleigh surface waves in linear thermoelastic materials with voids. They showed that the transverse wave is not influenced by the thermal and void effects at all, while the dilatational waves are coupled with thermal and porous fields. Svanadze [28] developed a potential method in the linear theory of viscoelastic materials with voids. Bhagwan and Tomar [37] studied reflection and transmission of plane dilatational wave through an interface between an elastic solid and a thermo-viscoelastic solid half-space with voids. Chandrasekharaiah [17] studied the wave like disturbances as second sound. Recently, D'Apice and Chirit a [38] analyzed the behaviour of plane harmonic waves in the linear thermo-viscoelastic materials with voids within the context of the theory of Iesan [1]. They showed that there may exist five basic waves consisting of three dilatational waves and two shear waves. The additional transverse wave exists only under a special circumstance. Both the shear waves are uncoupled, damped in time with decay rate depending only on the viscosity coefficients. The three dilatational waves are coupled and consist of a predominantly dilatational damped wave of KelvinVoigt viscoelasticity, the second is predominantly a wave carrying a change in the void volume fraction and the third takes the form of a standing thermal wave whose amplitude decays exponentially with 
time. Santra et al. [39] studied reflection and refraction due to incident $\mathrm{P}-$ and SV - waves at a plane interface between two dissimilar half spaces composed of generalized visco-thermoelastic materials. The generalization is considered under GL model of thermoelasticity and the effect of thermo-visco elasticity is noticed on various amplitude ratios corresponding to reflected and refracted waves at the interface.

In this paper, we have considered the coupled theory of thermoelasticity and its two generalizations due to Lord and Shulman [8] and Green and Lindsay [9]. The main differences between the considered generalized theories are as follows: (i) The Lord-Shulman theory modifies only the heat equation of the coupled theory, while the Green-Lindsay theory modifies both the constitutive equation and the energy equation. Consequently, the Lord-Shulman theory includes only one relaxation time, while the GreenLindsay theory involves two relaxation times. (ii) The heat equation of LST depends upon strain-velocity and strain-acceleration, whereas in GLT, heat equation depends only on strain-velocity. (iii) In the linearized case of GLT, the stresses must depend on temperature for the propagation of heat with finite speed, while in LST, this type of dependence on temperature is not necessary. In the present work, we have studied the propagation of plane time harmonic waves in an infinite thermo-viscoelastic material with voids within the context of generalized theories of thermo-elasticity [LST and GLT]. The relevant equations of motion and constitutive relations are modified from those already presented by Iesan [1]. It is found that three coupled dilatational waves and a lone shear wave can propagate with distinct speeds in an infinite thermo-viscoelastic medium with voids. Thermal and void properties are found to be the reason of appearance of additional dilatational waves and also responsible for the coupling among them. Some reduced cases of thermo-viscoelastic material with voids are discussed. The phase speeds, corresponding attenuation coefficients and energy flux carried along each wave under different theories considered are computed numerically and presented graphically. Coupling parameters among longitudinal waves have been looked into. Some concluding remarks are given in the end of the paper.

\section{Constitutive relations and equations}

Iesan [1] developed the constitutive relations and field equations for a linear homogeneous isotropic thermo-viscoelastic material with voids. Within the context of the Lord-Shulman and Green-Lindsay theories of thermoelasticity, the constitutive relations for uniform thermo-viscoelastic material with voids can be written as follows

$$
\begin{aligned}
& t_{i j}=\left(\lambda e_{r r}+b \phi+\lambda^{*} \dot{e}_{r r}+b^{*} \dot{\phi}-\beta \theta-\beta t_{l} \delta_{k 2} \dot{\theta}\right) \delta_{i j}+2 \mu e_{i j}+2 \mu^{*} \dot{e}_{i j}, \\
& H_{i}=\alpha \varphi_{, i}+\alpha^{*} \dot{\phi}_{, i}+\tau^{*} \theta_{, i}, \\
& g=-b e_{r r}-\gamma^{*} \dot{e}_{r r}-\xi \phi-\xi^{*} \dot{\phi}+m \theta \\
& \rho_{0} \eta=\beta e_{r r}+a \theta+m \phi+m t_{0} \delta_{k l} \dot{\phi}, \\
& Q_{i}+t_{0} \delta_{k l} \dot{Q}_{i}=k_{t} \theta_{, i}+\zeta \dot{\phi}_{, i},
\end{aligned}
$$

where $\lambda$ and $\mu$ are well known Lame's parameters; $b, \alpha, \xi$ and $\xi^{*}$ are the parameters corresponding to voids present in the medium; $\beta, \tau^{*}, m, k_{t}, \zeta$ and $a$ are the thermal parameters; $\lambda^{*}, \mu^{*}, b^{*}, \alpha^{*}$ and $\gamma^{*}$ are the viscoelastic parameters; $t_{i j}$ is the stress tensor; $e_{i j}$ is the strain tensor; $H_{i}$ is the equilibrated stress per unit area; $g$ is the equilibrated body force; $\eta$ is the entropy per unit mass; $Q_{i}$ is the heat flux vector and $\rho_{0}$ is the density of the medium. The entity $e_{r r}=u_{i, i}$ represents the dilatation, $u_{i}\left(x_{i}, t\right)$ are the components of 
displacement vector $\boldsymbol{u}, \phi$ is the change in void volume fraction, $\theta$ is the change in temperature from the constant ambient temperature $T_{0}$ and $\delta_{i j}$ is the Kronecker delta. A comma (,) before the subscripted index denotes the spatial partial derivative, while the dot over a symbol denotes the temporal derivative. Here, $t_{0}$ and $t_{1}$ are the relaxation times and the subscript $k$ identifies the different theories of thermo-elasticity. The case $k=1$ corresponds to LST and the case $k=2$ corresponds to GLT. When both $t_{0}$ and $t_{1}$ vanish, it corresponds to $\mathrm{CT}$ of thermoelasticity.

Within the context of generalized theories of thermoelasticity, the equations of motion for thermoviscoelastic material with voids can be written as

$$
\begin{aligned}
& \mu_{0} \nabla^{2} u_{i}+\left(\lambda_{0}+\mu_{0}\right) u_{j, j i}+b_{0} \phi_{, i}-\beta \tau_{l} \theta_{, i}=\rho_{0} \ddot{u}_{i}, \\
& \alpha_{0} \phi_{, i i}-v_{0} u_{j, j}-\xi_{0} \phi+\Gamma_{l} \theta=\rho_{0} \kappa \ddot{\phi}, \\
& c \tau_{0} \dot{\theta}+\beta T_{0} \tau_{0} \dot{u}_{i, i}-\Gamma_{2} \phi=k_{t} \nabla^{2} \theta,
\end{aligned}
$$

where

$$
\begin{aligned}
& \mu_{0}=\mu+\mu^{*} \frac{\partial}{\partial t}, \quad \lambda_{0}=\lambda+\lambda^{*} \frac{\partial}{\partial t}, \quad b_{0}=b+b^{*} \frac{\partial}{\partial t}, \quad \alpha_{0}=\alpha+\alpha^{*} \frac{\partial}{\partial t}, \\
& v_{0}=b+\gamma^{*} \frac{\partial}{\partial t}, \quad \xi_{0}=\xi+\xi^{*} \frac{\partial}{\partial t}, \quad \Gamma_{1}=\tau^{*} \nabla^{2}+m, \quad \Gamma_{2}=\left(\zeta \nabla^{2}-m T_{0} \tau_{0}\right) \frac{\partial}{\partial t}, \\
& \tau_{0}=1+\delta_{1 k} t_{0} \frac{\partial}{\partial t}, \quad \tau_{1}=1+\delta_{2 k} t_{1} \frac{\partial}{\partial t}, \quad c=a T_{0} .
\end{aligned}
$$

We define the following non-dimensional quantities as

$$
x_{i}^{\prime}=\frac{x_{i}}{l_{0}}, \quad u_{i}^{\prime}=\frac{u_{i}}{l_{0}}, \quad t^{\prime}=\frac{c_{0} t}{l_{0}}, \quad T^{\prime}=\frac{\theta}{T_{0}}
$$

where $l_{0}$ and $c_{0}$ denote respectively the standard length and standard velocity. With the help of these nondimensional quantities, Eqs (2.6) - (2.8) can be written, after suppressing the primes, as

$$
\begin{aligned}
& \mu_{1} \nabla^{2} u_{i}+\mu_{i}^{*} \nabla^{2} \dot{u}_{i}+\left(\lambda_{l}+\mu_{l}\right) u_{j, j i}+\left(\lambda_{l}^{*}+\mu_{1}^{*}\right) \dot{u}_{j, j i}+b_{l} \phi_{, i}+b_{1}^{*} \dot{\phi}_{, i}-\beta_{1} T_{, i}-\beta_{2} \delta_{2 k} \dot{T}_{, i}=\ddot{u}_{i}, \\
& \alpha_{l} \nabla^{2} \phi+\alpha_{l}^{*} \nabla^{2} \dot{\phi}-b_{l} u_{j, j}-\gamma_{l}^{*} \dot{u}_{j, j}-\xi_{l} \phi-\xi_{l}^{*} \dot{\phi}+\tau_{l}^{*} \nabla^{2} T+m_{l} T=r_{l} \ddot{\phi} \\
& \left(1+n_{1} \delta_{1 k} \frac{\partial}{\partial t}\right) \dot{T}+\left(\frac{\beta_{1}}{\tau_{3}}+\frac{\beta_{1}}{\tau_{2}} \delta_{1 k} \frac{\partial}{\partial t}\right) \dot{u}_{i, i}+\left(\frac{m_{1}}{\tau_{3}}+\frac{m_{1}}{\tau_{2}} \delta_{1 k} \frac{\partial}{\partial t}-\zeta_{l} \nabla^{2}\right) \dot{\phi}=k_{l} \nabla^{2} T
\end{aligned}
$$

where

$$
\begin{aligned}
& \lambda_{1}=\frac{\lambda}{\rho_{0} c_{0}^{2}}, \quad \lambda_{1}^{*}=\frac{\lambda^{*}}{\rho_{0} c_{0} l_{0}}, \quad \mu_{1}=\frac{\mu}{\rho_{0} c_{0}^{2}}, \quad \mu_{1}^{*}=\frac{\mu^{*}}{\rho_{0} c_{0} l_{0}}, \quad b_{1}=\frac{b}{\rho_{0} c_{0}^{2}}, \\
& b_{1}^{*}=\frac{b^{*}}{\rho_{0} c_{0} l_{0}}, \quad \beta_{1}=\frac{\beta T_{0}}{\rho_{0} c_{0}^{2}}, \quad \beta_{2}=\frac{\beta T_{0} t_{1}}{\rho_{0} c_{0} l_{0}}, \quad k_{1}=\frac{k_{t}}{c c_{0} l_{0}}, \quad n_{1}=\frac{t_{0} c_{0}}{l_{0}}, \quad r_{1}=\frac{\kappa}{l_{0}^{2}},
\end{aligned}
$$




$$
\begin{aligned}
& \zeta_{1}=\frac{\zeta}{c c_{0} T_{0} l_{0}}, \quad m_{1}=\frac{T_{0} m}{\rho_{0} c_{0}^{2}}, \quad \tau_{2}=\frac{T_{0} l_{0} c}{\rho_{0} c_{0}^{3} t_{0}}, \quad \tau_{3}=\frac{T_{0} c}{\rho_{0} c_{0}^{2}}, \quad \alpha_{1}=\frac{\alpha}{\rho_{0} c_{0}^{2} l_{0}^{2}}, \\
& \xi_{1}=\frac{\xi}{\rho_{0} \mathrm{c}_{0}^{2}}, \quad \tau_{1}^{*}=\frac{\mathrm{T}_{0} \tau^{*}}{\rho_{0} \mathrm{c}_{0}^{2} 1_{0}^{2}}, \quad \gamma_{1}^{*}=\frac{\gamma^{*}}{\rho_{0} \mathrm{c}_{0} \mathrm{l}_{0}}, \quad \xi_{1}^{*}=\frac{\xi^{*}}{\rho_{0} \mathrm{c}_{0} \mathrm{l}_{0}}, \quad \alpha_{1}^{*}=\frac{\alpha^{*}}{\rho_{0} \mathrm{c}_{0} 1_{0}^{3}} .
\end{aligned}
$$

In view of the second law of thermodynamics, the Cauchy-Duhem inequality provides the following restrictions among various material moduli

$$
3 \lambda^{*}+2 \mu^{*} \geq 0, \quad \mu^{*} \geq 0, \quad \alpha^{*} \geq 0, \quad k_{t} \geq 0, \quad \xi^{*} \geq 0, \quad T_{0}\left(\tau^{*}+\frac{1}{T_{0}} \zeta\right)^{2} \leq 4 \alpha^{*} k_{t} .
$$

Introduce the scalar potential $p$ and vector potential $\boldsymbol{\Psi}$ through Helmholtz decomposition theorem as

$$
\boldsymbol{u}=\nabla p+\nabla \times \Psi, \quad \nabla \cdot \Psi=0 .
$$

Here, the potential $p$ corresponds to the dilatational wave potential and the potential $\boldsymbol{\Psi}$ corresponds to the shear wave potential. Plugging (2.12) into Eqs (2.9)-(2.11), we obtain

$$
\begin{aligned}
& \nabla\left(c_{1}^{\prime 2} \nabla^{2} p+c_{2}^{\prime 2} \phi-c_{3}^{\prime 2} T-\ddot{p}\right)+\nabla \times\left(c_{12}^{\prime 2} \nabla^{2} \Psi-\ddot{\Psi}\right)=0, \\
& k_{1} \nabla^{2} T-c_{9}^{\prime 2} \dot{\phi}+\zeta_{1} \nabla^{2} \dot{\phi}-c_{10}^{\prime 2} \nabla^{2} \dot{p}-c_{11}^{\prime 2} \dot{T}=0, \\
& \left(c_{4}^{\prime 2}-c_{7}^{\prime 2}\right) \phi-c_{5}^{\prime 2} \nabla^{2} p+c_{6}^{\prime 2} T+c_{8}^{\prime 2} \nabla^{2} T-\ddot{\phi}=0,
\end{aligned}
$$

where

$$
\begin{aligned}
& c_{1}^{\prime 2}=\left(\lambda_{1}+\lambda_{1}^{*} \frac{\partial}{\partial t}\right)+2\left(\mu_{1}+\mu_{1}^{*} \frac{\partial}{\partial t}\right), \quad c_{2}^{\prime 2}=b_{1}+b_{1}^{*} \frac{\partial}{\partial t}, \quad c_{3}^{\prime 2}=\beta_{1}+\beta_{2} \delta_{2 k} \frac{\partial}{\partial t}, \\
& c_{4}^{\prime 2}=\frac{1}{r_{l}}\left(\alpha_{1}+\alpha_{1}^{*} \frac{\partial}{\partial t}\right), \quad c_{5}^{\prime 2}=\frac{1}{r_{l}}\left(b_{1}+\gamma_{1}^{*} \frac{\partial}{\partial t}\right), \quad c_{6}^{\prime 2}=\frac{m_{1}}{r_{1}}, \quad c_{7}^{\prime 2}=\frac{1}{r_{l}}\left(\xi_{1}+\xi_{1}^{*} \frac{\partial}{\partial t}\right), \quad c_{8}^{\prime 2}=\frac{\tau_{1}^{*}}{r_{l}}, \\
& c_{9}^{\prime 2}=\frac{m_{1}}{\tau_{3}}+\frac{m_{l} \delta_{1 k}}{\tau_{2}} \frac{\partial}{\partial t}, \quad c_{10}^{\prime 2}=\frac{\beta_{1}}{\tau_{3}}+\frac{\beta_{1} \delta_{1 k}}{\tau_{2}} \frac{\partial}{\partial t}, \quad c_{11}^{\prime 2}=1+n_{1} \delta_{1 k} \frac{\partial}{\partial t}, \quad c_{12}^{\prime 2}=\mu_{1}+\mu_{1}^{*} \frac{\partial}{\partial t} .
\end{aligned}
$$

Equation (2.13) yields

$$
\begin{aligned}
& c_{1}^{\prime 2} \nabla^{2} p+c_{2}^{\prime 2} \phi-c_{3}^{\prime 2} T=\ddot{p}, \\
& c_{12}^{\prime 2} \nabla^{2} \boldsymbol{\Psi}=\ddot{\boldsymbol{\Psi}} .
\end{aligned}
$$

We note that Eq.(2.17) is uncoupled in the potential $\Psi$, while Eqs (2.14) to (2.16) are coupled in the potentials $p, T$ and $\phi$. In the following section, we shall investigate the kind of waves carried along these equations. 


\section{Time harmonic waves}

For time harmonic wave motion, we shall take the form of various potentials as

$$
\{p, \phi, T, \Psi\}=\{\bar{p}, \bar{\phi}, \bar{T}, \bar{\Psi}\} \exp (\iota \omega t)
$$

where $\bar{p}, \bar{\phi}, \bar{T}$ and $\bar{\Psi}$ are functions of spatial co-ordinates only; $\omega$ and $t$ are respectively the nondimensional frequency and non-dimensional time. The non-dimensional time variable has been defined earlier; while the non-dimensional frequency is defined as $\omega^{\prime}=\frac{l_{0} \omega}{c_{0}}$ (later the prime has been dropped). Inserting the expressions of $p, \phi$ and $T$ from Eq.(3.1) into Eqs (2.14) - (2.16) and suppressing over bars, we obtain

$$
\begin{aligned}
& \left(c_{1}^{2} \nabla^{2}+\omega^{2}\right) p+c_{2}^{2} \phi-c_{3}^{2} T=0, \\
& \left(c_{4}^{2} \nabla^{2}+\omega^{2}-c_{7}^{2}\right) \phi-c_{5}^{2} \nabla^{2} p+\left(c_{8}^{2} \nabla^{2}+c_{6}^{2}\right) T=0, \\
& \left(k_{1} \nabla^{2}-\imath \omega+c_{11}^{2} \omega^{2}\right) T-\imath \omega c_{10}^{2} \nabla^{2} p+\imath \omega\left(\zeta_{I} \nabla^{2}-c_{9}^{2}\right) \phi=0,
\end{aligned}
$$

where

$$
\begin{aligned}
& c_{1}^{2}=\lambda_{1}+2 \mu_{1}+1 \omega\left(\lambda_{1}^{*}+2 \mu_{1}^{*}\right), \quad c_{2}^{2}=b_{1}+1 \omega b_{1}^{*}, \quad c_{3}^{2}=\beta_{1}+1 \omega \beta_{2} \delta_{2 k}, \\
& c_{4}^{2}=\frac{1}{r_{1}}\left(\alpha_{1}+1 \omega \alpha_{1}^{*}\right), \quad c_{5}^{2}=\frac{1}{r_{1}}\left(b_{1}+1 \omega \gamma_{1}^{*}\right), \quad c_{6}^{2}=\frac{m_{1}}{r_{1}}, \quad c_{7}^{2}=\frac{1}{r_{1}}\left(\xi_{1}+1 \omega \xi_{1}^{*}\right), \\
& c_{8}^{2}=\frac{\tau_{1}^{*}}{r_{1}}, \quad c_{9}^{2}=m_{1}\left(\frac{1}{\tau_{3}}+\frac{\delta_{1 k}}{\tau_{2}} \imath \omega\right), \quad c_{10}^{2}=\beta_{1}\left(\frac{1}{\tau_{3}}+\frac{\delta_{1 k}}{\tau_{2}} 1 \omega\right), \quad c_{11}^{2}=1 \omega \delta_{1 k} n_{1} .
\end{aligned}
$$

The quantities $T$ and $\phi$ are connected with $p$ through the coupling parameters $G$ and $F$ given by

$$
\{T, \phi\}=\{G, F\} p,
$$

where

$$
\begin{aligned}
& G, F=\frac{A_{2,3} \nabla^{4}+\omega^{2} B_{2,3} \nabla^{2}}{A_{1} \nabla^{4}+\omega^{2} B_{1} \nabla^{2}+\omega^{4} C Z+\omega^{6} C_{1}}, \\
& A_{1}=1 \omega \zeta_{1} c_{8}^{2}-k_{1} c_{4}^{2}, \quad A_{2}=1 \omega\left(c_{4}^{2} c_{10}^{2}-\zeta_{1} c_{5}^{2}\right), \quad A_{3}=-1 \omega c_{8}^{2} c_{10}^{2}+k_{1} c_{5}^{2}, \\
& B_{1}=\frac{1}{\omega^{2}}\left[-\omega^{2}\left(k_{1}+c_{4}^{2} c_{11}^{2}\right)+1 \omega\left(c_{4}^{2}-c_{8}^{2} c_{9}^{2}+\zeta_{1} c_{6}^{2}\right)+k_{1} c_{7}^{2}\right], \quad B_{2}=\frac{1}{\omega}\left(c_{5}^{2} c_{9}^{2}+\omega^{2} c_{10}^{2}-c_{7}^{2} c_{10}^{2}\right), \\
& B_{3}=\frac{-1}{\omega}\left(c_{6}^{2} c_{10}^{2}+c_{5}^{2}\right)+c_{5}^{2} c_{11}^{2}, \quad C_{1}=\frac{1}{\omega^{3}}\left(\omega^{2}-c_{7}^{2}-c_{6}^{2} c_{9}^{2}\right)+\frac{1}{\omega^{2}} c_{7}^{2} c_{11}^{2}-c_{11}^{2} .
\end{aligned}
$$

Using Eq.(3.5) in Eq.(3.2), we obtain 


$$
\left\{A \nabla^{6}+\omega^{2} B \nabla^{4}+\omega^{4} C \nabla^{2}+\omega^{6} C\right\} p=0
$$

where $\quad A=c_{1}^{2} A_{1}, \quad B=c_{1}^{2} B_{1}+A_{1}+\frac{c_{2}^{2}}{\omega^{2}} A_{3}-\frac{c_{3}^{2}}{\omega^{2}} A_{2}, \quad$ and $\quad C=c_{1}^{2} C_{1}+B_{1}+\frac{c_{2}^{2}}{\omega^{2}} B_{3}-\frac{c_{3}^{2}}{\omega^{2}} B_{2}$.

The corresponding auxiliary equation of (3.7) is a sixth degree equation, which on notating $\nabla^{2} \equiv Z$. can be written as

$$
A Z^{3}+\omega^{2} B Z^{2}+\omega^{4} C Z+\omega^{6} C=0
$$

The solution of Eq.(3.7) can be written in the form

$$
p=\sum_{i=1}^{3} p_{i}
$$

where the potential function $p_{i}(i=1,2,3)$ satisfies the following well-known Helmholtz equation

$$
\left(\nabla^{2}+\frac{\omega^{2}}{v_{i}^{2}}\right) p_{i}=0
$$

This explains the existence of three dilatational waves, say $P_{I}, P_{I I}$ and $P_{I I I}$ propagating with speeds $v_{I}$, $v_{2}$ and $v_{3}$, respectively. The speeds $v_{i}(i=1,2,3)$ are given by $v_{i}^{2}=-\omega^{2} Z_{i}^{-1}$, where $Z_{i}$ are the roots of Eq.(3.8).

Next, inserting $\boldsymbol{\Psi}$ from Eq.(3.1) into Eq.(2.17) and dropping over bars, we have

$$
\left(\nabla^{2}+\frac{\omega^{2}}{v_{4}^{2}}\right) \Psi=0, \quad v_{4}=\sqrt{\mu_{1}+1 \omega \mu_{1}^{*}} .
$$

This represents an equation of shear wave propagating with speed $v_{4}$ in a thermo-viscoelastic medium with voids. The expression of speed $v_{4}$ is non-dimensional and can be written as equal to $\frac{1}{c_{0}} \sqrt{\frac{\left(\mu+1 \omega \mu^{*}\right)}{\rho_{0}}}$, which matches with the speed of shear wave in a viscoelastic medium. Hence the thermal property and presence of voids in the medium are unable to alter the speed of shear wave. Thus, we note that there exist four waves consisting of three dilatational waves and one shear wave propagating with distinct speeds. It can be seen that the speeds of all these propagating waves are complex valued and depend upon the frequency parameter, indicating that the relevant waves are dispersive and attenuated. The speeds of three dilatational waves can be determined from Eq.(3.7) by following the same procedure as adopted in Tomar et al. [32]. The phase speeds $V_{j} ;(j=1,2,3)$ and the corresponding attenuation coefficients $A_{j}$ of all the existing waves can be calculated from the formulae

$$
V_{j}=\frac{\left(\mathfrak{R}\left(v_{j}\right)\right)^{2}+\left(\mathfrak{I}\left(v_{j}\right)\right)^{2}}{\mathfrak{R}\left(v_{j}\right)}, \quad A_{j}=\frac{-\mathfrak{I}\left(v_{j}\right) \omega}{\left(\mathfrak{R}\left(v_{j}\right)\right)^{2}+\left(\mathfrak{I}\left(v_{j}\right)\right)^{2}} .
$$


Here, the symbols $\mathfrak{R}()$ and $\mathfrak{I}()$ denote respectively the real and imaginary parts. Now, using Eqs (3.9) and (3.10) in Eq.(3.5), we obtain

$$
\{T, \phi\}=\sum\left\{G_{i}, F_{i}\right\} p_{i}
$$

where the quantity $G_{i}$ represents the coupling between $T$ and $p_{i}$, while the quantity $F_{i}$ represents the coupling between $\phi$ and $p_{i}$. Using Eq.(3.10) in Eq.(3.6), these coupling parameters are given by the formulae

$$
G_{i}=\frac{A_{2}-B_{2} v_{i}^{2}}{A_{1}-B_{1} v_{i}^{2}+C_{1} v_{i}^{4}}, \quad F_{i}=\frac{A_{3}-B_{3} v_{i}^{2}}{A_{1}-B_{1} v_{i}^{2}+C_{1} v_{i}^{4}}
$$

In the following section, we shall analyze the behaviour of these coupling parameters.

\section{Behaviour of coupling parameters}

As noted above that the three dilatational waves are coupled under three considered theories of thermoelasticity. Here, we wish to investigate under what situation these dilatational waves are uncoupled? From the expressions of coupling coefficients $G_{i}$ and $F_{i}$ it is clear that these coefficients must vanish if the expressions of quantities $A_{2}, B_{2}, A_{3}$ and $B_{3}$ vanish. Now the quantities $A_{2}$ and $B_{2}$ will vanish if the thermal parameters are absent from the medium, while the quantities $A_{3}$ and $B_{3}$ will vanish if both thermal and void parameters are absent from the medium. Hence, we can say that the dilatational wave will be uncoupled when thermal waves and voids are absent from the medium. In this case only one dilatational wave will survive as can be seen in the subsequent section. In other words, we can say that the additional dilatational waves appear due to the presence of thermal waves and voids in the medium, which are also responsible for the coupling among them. For the three dilatational waves to be coupled, both the coupling coefficients must not vanish simultaneously. This is possible when the medium is equipped with thermal waves and voids. The behaviour of coupling parameters versus frequency has been investigated numerically for a particular model. For non-vanishing values of frequency, we found that coupling parameters never vanish under any of the theories of thermoelasticity considered, however at zero frequency, both the coupling parameters vanish as can be verified from Eq.(3.14).

\section{Special cases}

In this section, we shall reduce the speed of the propagating plane waves within the context of different theories of thermoelasticity. Recalling that the value $k=1$ corresponds to the Lord-Shulman theory, and the value $k=2$ corresponds to the Green-Lindsay theory, while the value $t_{0}=t_{l}=0$ corresponds to the coupled theory of thermoelasticity.

\subsection{Lord-Shulman theory}

When $\mathrm{k}=1$, the symbols given above immediately after Eq.(3.4) shall remain same, except those containing Kronecker delta, which reduce to

$$
c_{3}^{2}=\beta_{1}, \quad c_{9}^{2}=\frac{m_{1}}{\tau_{3}}+\frac{m_{1}}{\tau_{2}} 1 \omega, \quad c_{10}^{2}=\frac{\beta_{1}}{\tau_{3}}+\frac{\beta_{1}}{\tau_{2}} \imath \omega, \quad c_{11}^{2}=1 \omega n_{1}
$$


With these values of various symbols, one can find the speeds of various existing waves from formulae (3.12) within the context of LST.

\subsection{Green-Lindsay theory}

When $k=2$, the various notations immediately after Eq.(3.4) will remain same, except those containing Kronecker delta, which reduce to

$$
c_{3}^{2}=\beta_{1}+1 \omega \beta_{2}, \quad c_{9}^{2}=\frac{m_{1}}{\tau_{3}}, \quad c_{10}^{2}=\frac{\beta_{1}}{\tau_{3}}, \quad c_{11}^{2}=0
$$

With these values of various symbols, one can find the speeds of various existing waves from formulae (3.12) within the context of GLT.

\subsection{Coupled theory}

Here, we take $t_{0}=t_{1}=0$. In this case, the affected quantities given after Eq.(3.4) take the form

$$
c_{3}^{2}=\beta_{1}, \quad c_{9}^{2}=\frac{m_{1}}{\tau_{3}}, \quad c_{10}^{2}=\frac{\beta_{1}}{\tau_{3}}, \quad c_{11}^{2}=0,
$$

and the speeds of various waves can be calculated as done under other theories of thermoelasticity. To reduce the speed of existing waves in a classical elastic medium and in an elastic medium with voids, we shall take the relaxation times $t_{0}=t_{1}=0$. With these considerations, one gets the same equations and expressions as obtained by Tomar et al. [34] for the corresponding problem.

\section{Energy of waves}

Following Achenbach [40], the rate of average energy transmission per unit area $\left(E^{*}\right)$ at $y=0$ is given by

$$
E^{*}=\mathfrak{R}\left(t_{z z} \dot{u}_{z}\right)+\mathfrak{R}\left(t_{x z} \dot{u}_{x}\right)+\mathfrak{R}\left(H_{z} \dot{\phi}\right)+\mathfrak{R}\left(Q_{z} \dot{\theta}\right)
$$

Using the result $\mathfrak{R}(f) \Re(g)=\frac{1}{2} \mathfrak{R}(f \bar{g})$, over bar being the complex conjugate, the above expression reduces to

$$
E^{*}=\frac{1}{2} \mathfrak{R}\left(t_{z z} \overline{\dot{u}}_{z}\right)+\frac{1}{2} \mathfrak{R}\left(t_{x z} \overline{\dot{u}}_{x}\right)+\frac{1}{2} \mathfrak{R}\left(H_{z} \overline{\dot{\phi}}\right)+\Re\left(Q_{z} \overline{\dot{\theta}}\right)
$$

This formula will help us to determine the average energy carried along dilatational and shear waves. The various potential functions $p, \phi, \theta$ and $\boldsymbol{\Psi}$ for plane waves propagating in the $x-z$ plane in a thermoviscoelastic medium with voids can be written as

$$
p_{i}(x, z, t)=A_{0} \exp \left\{1 k_{i}\left(x \sin \theta_{0}+z \cos \theta_{0}+\omega t\right)\right\},
$$




$$
\begin{aligned}
& T(x, z, t)=\sum_{i=1}^{3} G_{i} A_{0} \exp \left\{\mathrm{l} k_{i}\left(x \sin \theta_{0}+z \cos \theta_{0}+\omega t\right)\right\}, \\
& \phi(x, z, t)=\sum_{i=1}^{3} F_{i} A_{0} \exp \left\{\mathrm{l} k_{i}\left(x \sin \theta_{0}+z \cos \theta_{0}+\omega t\right)\right\}, \\
& \psi(x, z, t)=\boldsymbol{B}_{0} \exp \left\{\mathrm{l} k_{4}\left(x \sin \theta_{0}+z \cos \theta_{0}+\omega t\right)\right\},
\end{aligned}
$$

the wave normal of the wave makes angle $\theta_{0}$ with the positive direction of the $z$-axis. The $k_{j} ; \quad(j=1,2,3,4)$ and $\omega$ are the wavenumber and angular frequency, respectively, for the propagating waves; $A_{0}$ is a scalar amplitude, $\boldsymbol{B}_{0}$ is constant vector amplitude, the quantities $G_{i}$ and $F_{i}$ represent the coupling parameters given earlier in Eq.(3.14). Since the wave speeds are complex in nature, therefore for real valued frequency, the wavenumber will be complex.

Following Borcherd [41], the complex wavenumber $k_{j}$ written in terms of propagation vector $\boldsymbol{P}_{j}$ and attenuation vector $\boldsymbol{A}_{j}$ of the general type of inhomogeneous plane wave travelling in a dissipative medium, is given by

$$
k_{j}=\left|P_{j}\right| \sin \theta_{0}-1\left|A_{j}\right| \sin \left(\theta_{0}-\gamma_{j}\right), \quad(j=1,2,3,4),
$$

where $\gamma_{j}$ is the angle between the propagation vector $\boldsymbol{P}_{j}$ and the attenuation vector $\boldsymbol{A}_{j}$. The modulus of $\boldsymbol{P}_{j}$ and $\boldsymbol{A}_{j}$ are given by

$$
\begin{aligned}
& \left|P_{j}\right|^{2}=\frac{1}{2}\left\{\mathfrak{R}\left(k_{p j}^{2}\right)+\sqrt{\left(\mathfrak{R}\left(k_{p j}^{2}\right)\right)^{2}+\frac{\left(\mathfrak{I}\left(k_{p j}^{2}\right)\right)^{2}}{\left(\cos \gamma_{j}\right)^{2}}}\right\}, \\
& \left|A_{j}\right|^{2}=\frac{1}{2}\left\{-\mathfrak{R}\left(k_{p j}^{2}\right)+\sqrt{\left(\mathfrak{R}\left(k_{p j}^{2}\right)\right)^{2}+\frac{\left(\mathfrak{I}\left(k_{p j}^{2}\right)\right)^{2}}{\left(\cos \gamma_{j}\right)^{2}}}\right\}, \quad k_{p j}=\frac{\omega}{v_{j}} .
\end{aligned}
$$

Now, making use of Eqs (6.3) - (6.6) and (2.1) - (2.5) in Eq.(6.2), we obtain the energy flux carried along three coupled dilatational waves and a lone shear wave under different theories. It is found that the expressions of energy carried along different dilatational waves under CT and GLT remain same, while they are different under LST. Since transverse wave is not influenced by thermal property of the medium, therefore, its energy expression will be invariant. Energy carried along $P_{I}, P_{I I}$ and $P_{I I I}$-waves can be obtained by putting index $l=1,2,3$ respectively as:

Under the Coupled Theory and Green-Lindsay Theory

$$
E_{l}^{*}=\frac{\omega k_{l}}{2 \sqrt{2}}\left[\left(\frac{\lambda}{2}+2 \mu\right) k_{l}^{2}-b F_{l}+\beta T_{0} G_{l}+\alpha F_{l}^{2}+k_{t} G_{l}^{2} T_{0}^{2}+\tau^{*} T_{0} G_{l} F_{l}\right] A_{0}^{2} .
$$


Under the Lord-Shulman Theory

$$
E_{l}^{*}=\frac{\omega k_{l}}{2 \sqrt{2}}\left[\left(\frac{\lambda}{2}+2 \mu\right) k_{l}^{2}-b F_{l}+\beta T_{0} G_{l}+\alpha F_{l}^{2}+k_{t} G_{l}^{2} T_{0}^{2}+\left(\tau^{*}+\omega^{2} t_{0}\right) T_{0} G_{l} F_{l}\right] A_{0}^{2} .
$$

And the energy carried along $S$-wave is given by

$$
E_{4}^{*}=-\frac{\lambda \omega k_{4}^{3}}{4 \sqrt{2}} B_{0}^{2}
$$

It can be seen that the energy fluxes of dilatational waves are functions of frequency, wavenumber, temperature field, coupling parameters and elastic properties of the medium. While energy expression of transverse wave does not depend on temperature field as was expected before. Moreover, the energy of each wave is proportional to the square of the amplitude of corresponding wave. It can be noticed that the energy expressions of dilatational waves heavily depend on coupling parameters.

\section{Numerical discussion}

To understand the behaviour of phase speeds of various existing waves and their corresponding energies under different theories of thermoelasticity, we have computed them for a copper material. The numerical values of relevant parameters for the copper material have been taken from Mukhopadhyay [42] and given in Tab.1.

\begin{tabular}{|c|c|c|c|c|c|}
\hline Symbol & Value & Unit & Symbol & Value & Unit \\
\hline$\lambda$ & $8.2 \times 10^{10}$ & dyn $\mathrm{cm}^{-2}$ & $\mu$ & $4.2 \times 10^{10}$ & dyn $\mathrm{cm}^{-2}$ \\
\hline $\bar{b}$ & $0.20 \times 10^{10}$ & dyn $\mathrm{cm}^{-2}$ & $a$ & $0.8 \times 10^{2}$ & dyn $\mathrm{cm}^{-20} \mathrm{C}^{-1}$ \\
\hline$\rho_{0}$ & 8954 & $\mathrm{gm} \mathrm{cm}^{-3}$ & $c_{0}$ & 1.0 & $\mathrm{~cm} \mathrm{~s}^{-1}$ \\
\hline$l_{0}$ & 1.0 & $\mathrm{~cm}$ & $\beta$ & $0.15 \times 10^{6}$ & dyn $\mathrm{cm}^{-20} \mathrm{C}^{-1}$ \\
\hline$\lambda^{*}$ & $0.45 \times 10^{9}$ & dyn s cm ${ }^{-2}$ & $\mu^{*}$ & $0.1 \times 10^{9}$ & dyn s ${ }^{0} \mathrm{C}^{-1}$ \\
\hline$b^{*}$ & $0.1 \times 10^{10}$ & dyn s cm ${ }^{-2}$ & $\alpha$ & $1.5 \times 10^{-5}$ & dyn s cm ${ }^{-2}$ \\
\hline$\xi$ & $1.5 \times 10^{10}$ & dyn $\mathrm{cm}^{-2}$ & $\alpha^{*}$ & $1.0 \times 10^{-4}$ & dyn s \\
\hline$m$ & $0.2 \times 10^{6}$ & dyn $\mathrm{cm}^{-20} \mathrm{C}^{-1}$ & $T_{0}$ & 300 & $\mathrm{~K}$ \\
\hline$\tau^{*}$ & $0.01 \times 10^{6}$ & $\operatorname{dyn}{ }^{0} \mathrm{C}^{-1}$ & $\gamma^{*}$ & $0.2 \times 10^{9}$ & dyn s cm ${ }^{-2}$ \\
\hline$\xi^{*}$ & $1.0 \times 10^{6}$ & dyn s cm ${ }^{-2}$ & $\kappa$ & $0.15 \times 10^{-10}$ & $\mathrm{~cm}^{2}$ \\
\hline$k_{t}$ & $0.1 \times 10^{3}$ & dyn $\mathrm{s}^{-10} \mathrm{C}^{-1}$ & $\zeta$ & $0.1 \times 10^{6}$ & dyn \\
\hline
\end{tabular}

Table 1. Numerical values of relevant parameters.

The phase speeds and corresponding attenuation coefficients of three dilatational waves, namely $P_{I}, P_{I I}$ and $P_{I I I}$ and a lone shear wave are computed and depicted graphically with respect to frequency through Figs 1 and 2. Note that in all the figures drawn, the blue, black and red colour curves correspond to LST, CT and GLT, respectively. 

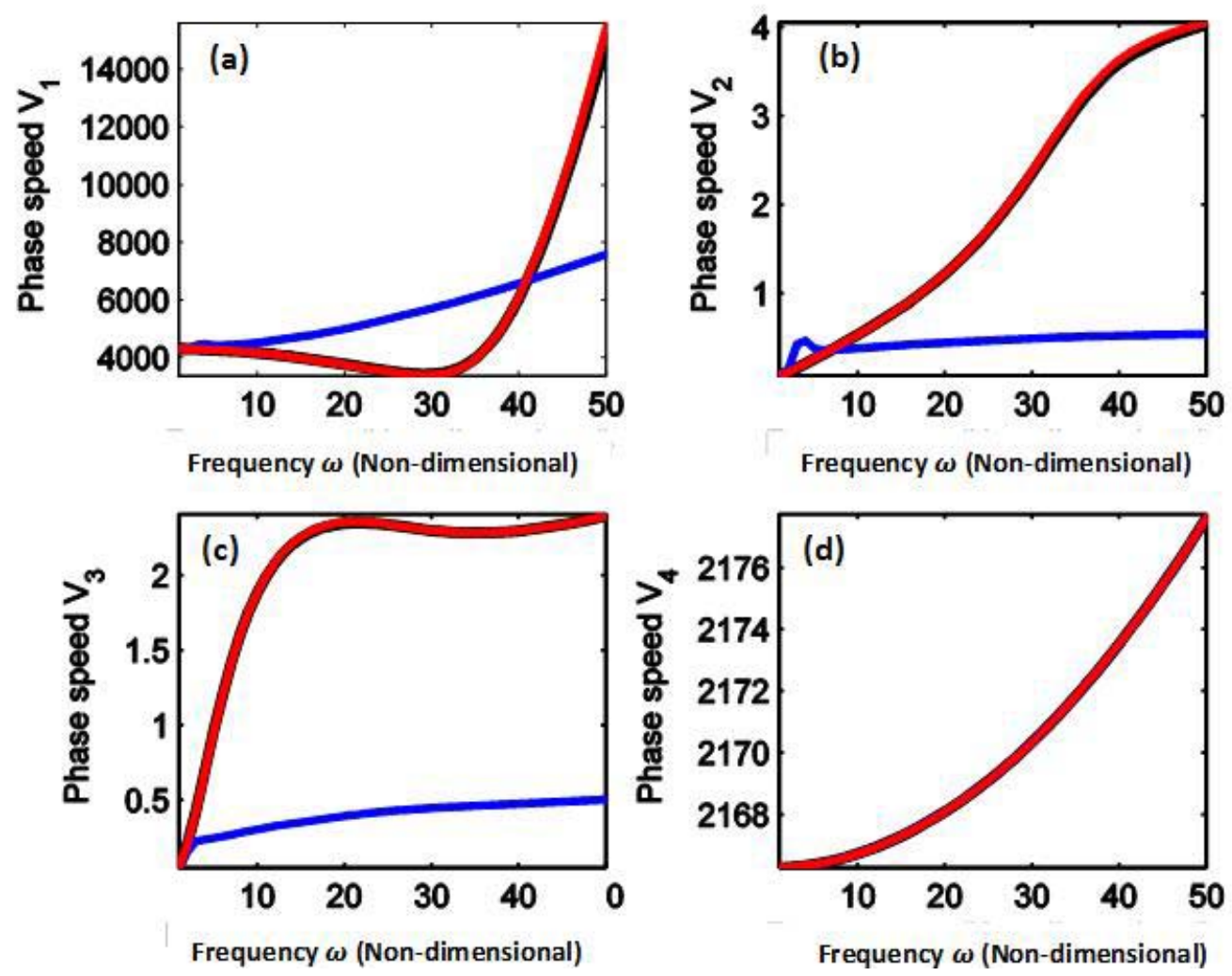

Fig.1. Behaviour of phase speeds of dilatational and shear waves under CT, GLT and LST.

Figure 1a depicts the variation of phase speed of $\mathrm{P}_{\mathrm{I}}$-wave versus frequency parameter under three theories of thermoelasticity considered. We notice from this figure that the phase speed of $P_{I}$ - wave is continuously increasing with frequency parameter under LST, while under CT and GLT, the phase speed of this wave, first decreases with an increase of frequency and then increases a little bit faster with a further increase of frequency parameter. Figure $1 \mathrm{~b}$ clearly depicts that the phase speed of $P_{I I}$-wave is more under LST near zero frequency $(0<\omega<6)$ than CT \& GLT, while in the remaining range of frequency, i.e., beyond $\omega>6$, the phase speed under CT \& GLT is higher than that of under LST. Note that in this range, the phase speed of $P_{I I}$ - wave under LST bears almost a constant value. The small kink appearing in the phase speed curve under LST is due to the presence of relaxation time $t_{0}$. It has been noticed that this kink goes up and up with an increase of $t_{0}$. From Fig.1c, we see that the phase speed of $P_{I I I}$-wave increases with an increase in the frequency parameter. The phase speed of this wave under CT and GLT is almost same. The phase speed under LST increases relatively at a much slower rate than that of CT and GLT. Hence, the phase speed under LST is much less than that of under CT and GLT. Figure 1d depicts the behaviour of phase speed $v_{4}$ of shear wave against the frequency parameter. One can observe that there is no change in the phase speed of this wave under CT, LST and GLT as was expected before hand. This is because this wave is not influenced by the presence of thermal and void properties of the medium. From these figures, one can infer that the phase speeds of $P_{I}, P_{I I}$ and $P_{I I I}$-waves are in decreasing order. Moreover, the phase speed of shear wave is less than the phase speed of the fastest dilatational wave. This fact is, in general, true for most of the deformable elastic solid materials. It has been verified that all the phase speeds become almost constant at high frequency values. Thus, they do not tend to unlimited with unlimited increase of the frequency parameter. 

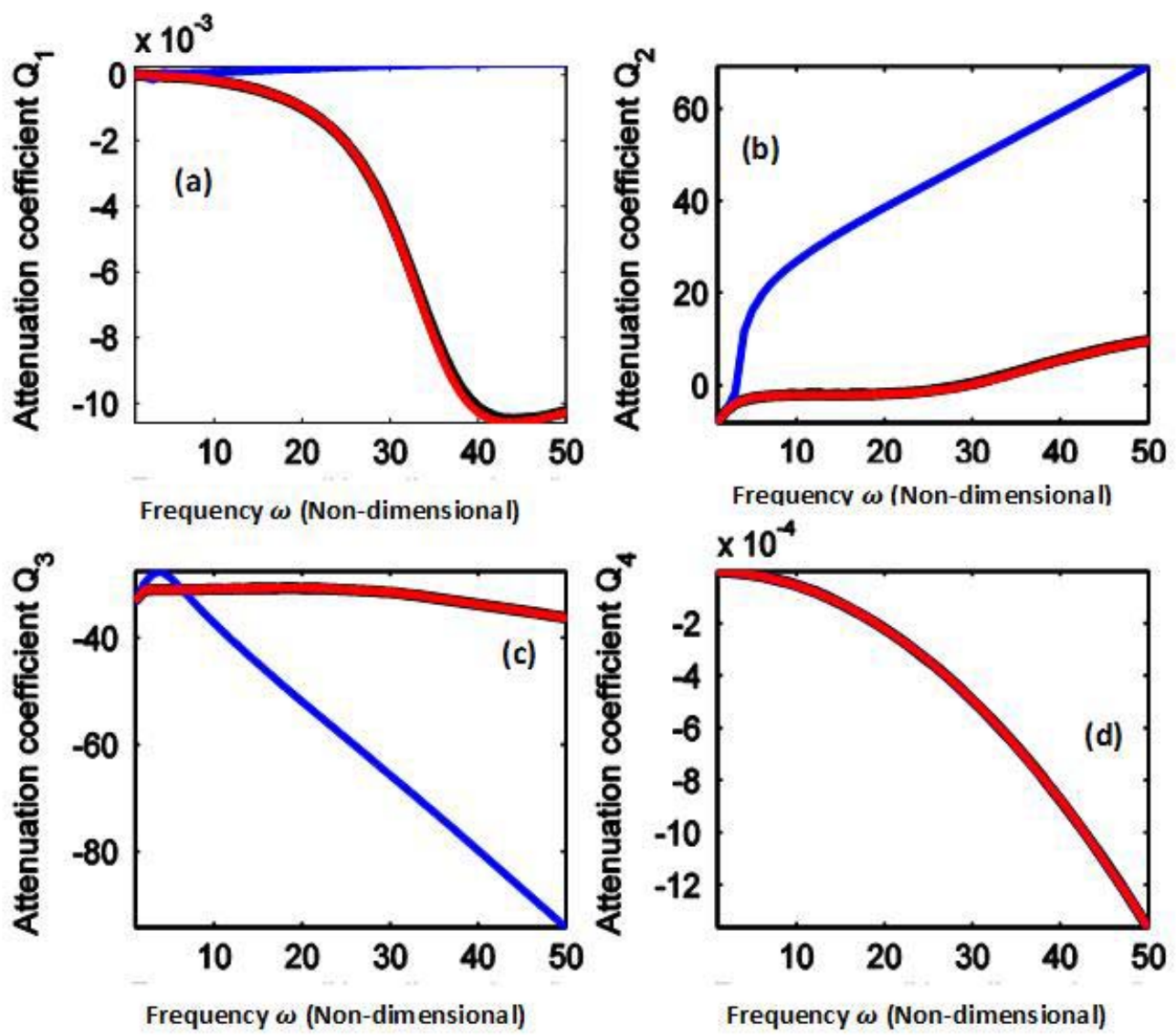

Fig.2. Behaviour of attenuation coefficients of dilatational and shear waves under CT, GLT and LST.

Figures $2 \mathrm{a}-\mathrm{c}$ depict the variation of attenuation coefficients of all the existing waves under different theories of thermoelasticity against the frequency parameter. From Fig.2a, we note that the attenuation of $P_{I}$ - wave is very small under CT, GLT and LST, which decreases with an increase of the frequency parameter under CT and GLT, while under LST, it remains almost constant. The attenuation coefficient of $P_{I I}$-wave increases with an increase of frequency under CT, LST and GLT. The rate of increase under CT and GLT is much smaller than that of under LST. But, the magnitude of attenuation coefficient of this wave is appreciable in comparison to that of all other waves. The behaviour of attenuation coefficient with frequency of $P_{I I I}$-wave is decreasing very fast under LST than that of under CT and GLT. From Fig.2d, we note that the attenuation coefficient of shear wave under LST, CT and GLT decreases monotonically with an increase in the frequency parameter. It is worth noting that the shear wave is least attenuating among the attenuations of all the other dilatational waves.

As we have noticed above that the three dilatational waves are coupled under each considered theories of thermoelasticity through the coupling coefficients $G_{i}$ and $F_{i},(i=1,2,3)$. Therefore, there are a total of six coupling coefficients under CT, GLT and LST, namely, $C_{11}, C_{12}, C_{21}, C_{22}, C_{31}$ and $C_{32}$. The behaviour of these coupling coefficients against the frequency parameter has been shown through Figs $3 \mathrm{a}-\mathrm{f}$. Basically, these coupling coefficients behave alike under CT and GLT, but differently under LST. From these figures, it is clear that both of the coupling coefficients cannot be zero simultaneously under considered theories of thermo-elasticity. Even at zero frequency, one of the coupling coefficients is non zero. This shows that the waves will remain coupled unless thermal and void parameters are absent from the medium. As these coupling coefficients depend upon thermal, viscoelastic and void parameters and these parameters 
have some non-zero values, therefore, they results in non-zero coupling coefficients. Non-vanishing value of coupling coefficients is the main cause of three coupled waves under CT, LST and GLT.
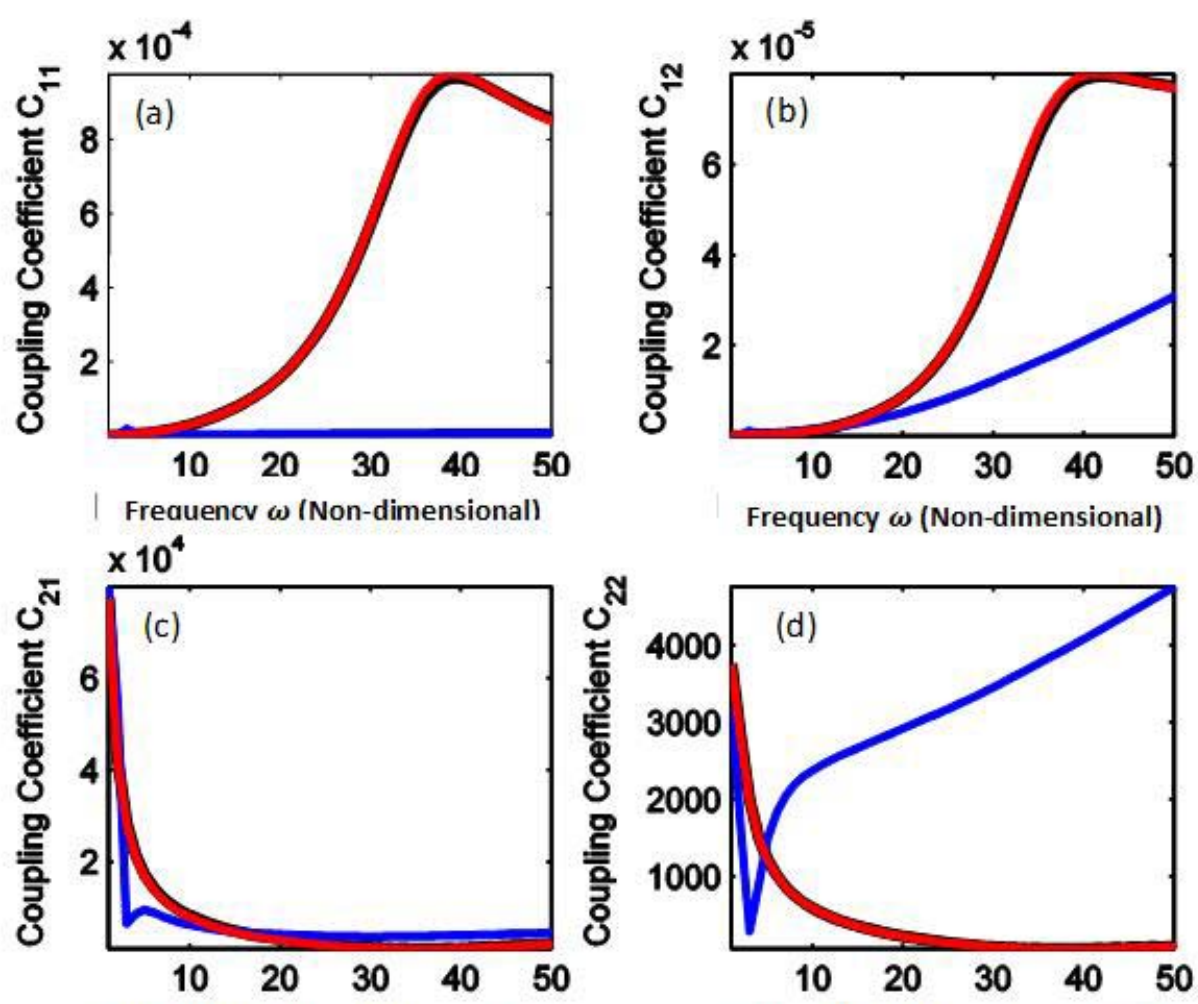

Frequency $\omega$ (Non-dimensional)

Frequency $\omega$ (Non-dimensional)
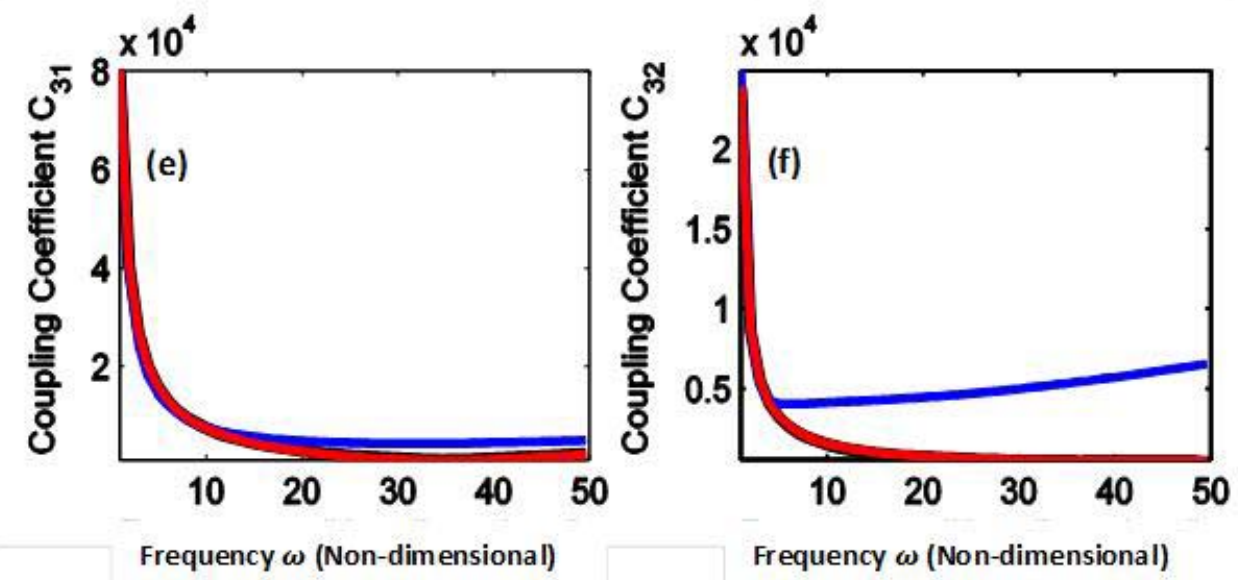

Fig.3. Behaviour of coupling coefficients of dilatational and shear waves under CT, GLT and LST.

Figures $4 \mathrm{a}-\mathrm{d}$ depict the energy flux carried along different waves under different theories of thermoelasticity. For computational purposes, the amplitudes of waves, that is, the quantities $A_{0}$ and $B_{0}$ have been taken unity. Firstly, we note that the energy flux of dilatational waves at different values of frequency is almost same under CT and GLT. Under LST, the energy flux of $P_{I}$ - wave increases smoothly, but slowly, with frequency, while for those of $P_{I I}$ - and $P_{I I I}$ - waves, it increases much faster with frequency. Energy flux of $P_{I I I}$ - wave under LST is relatively higher than those of other waves. The behaviour of energy 
flux of shear wave against frequency is same under GLT, LST and CT, and increases with an increase of the frequency parameter.
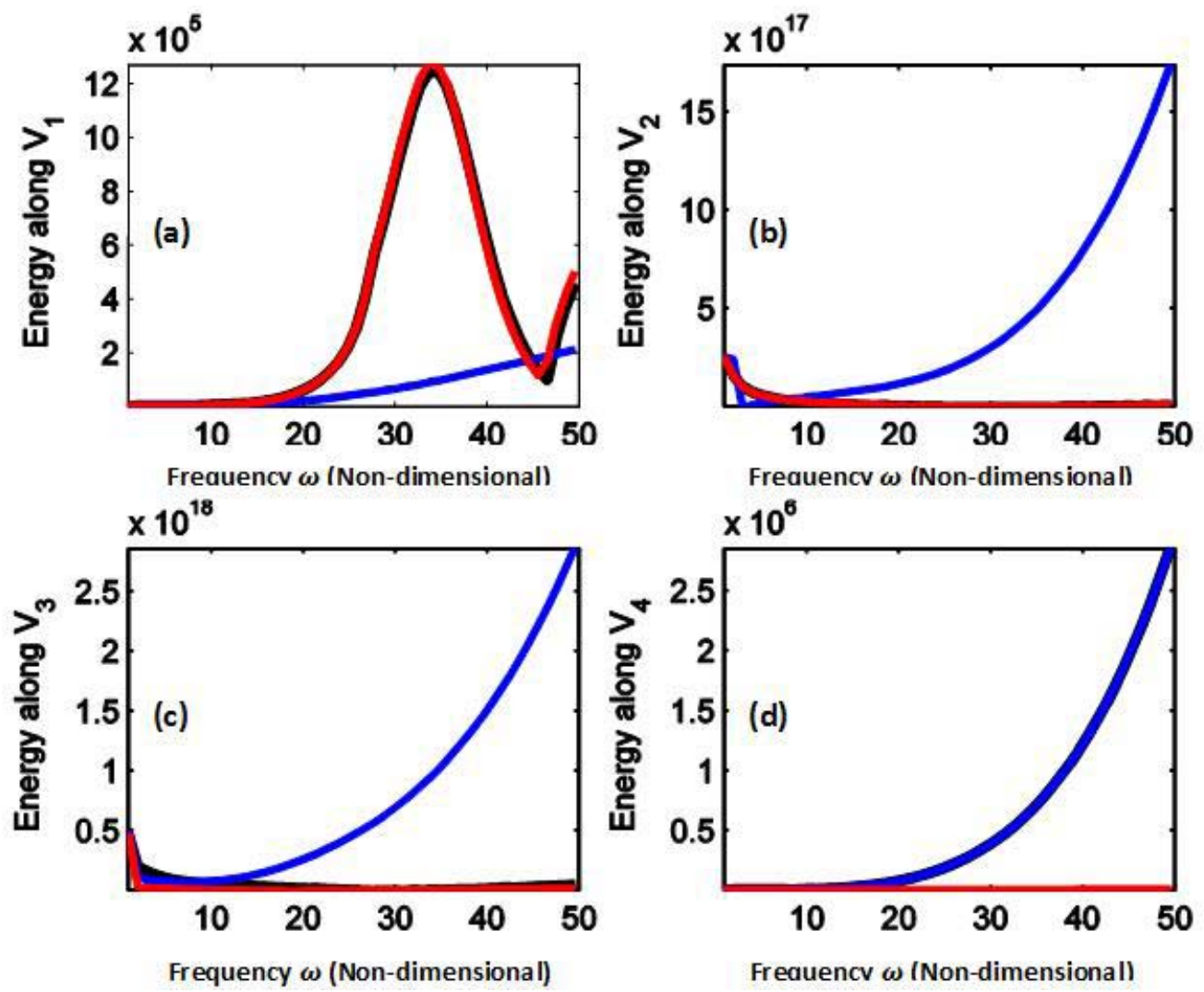

Fig.4. Behaviour of energy flux carried along dilatational and shear waves under CT, GLT and LST.

\section{Conclusion}

A mathematical method is presented to explore the effect of different theories of thermo-elasticity on time harmonic wave propagation through a thermo-viscoelastic material with voids. Constitutive equations and governing equations of a thermo-viscoelastic material with voids developed by Iesan [1] have been extended within the context of LST and GLT theories. For this purpose, two relaxation times have been introduced via the Kronecker delta into constitutive relations and equations, which can be easily reduced for corresponding equations of CT, LST and GLT. Under each of these theories, it has been found that three dilatational waves and a lone shear wave can travel in an infinite thermo-viscoelastic material with voids. The dilatational waves are found to be coupled, while the shear wave remains uncoupled. All the waves are attenuating and their corresponding phase speeds are dispersive in nature. It has also been shown that thermal and void parameters are responsible for the coupling of three dilatational waves. The phase speeds, corresponding attenuation coefficients and energy carried along each wave have been presented and computed numerically for a particular model. It has been found that phase speeds, attenuation coefficients and energy fluxes of all the waves are almost same under GLT and CT. But these are significantly influenced under LST. The thermal relaxation time of LST has a prominent effect on $P_{I I}$-wave. The kink in the phase velocity curve of $P_{I I}$-wave at certain frequency value arises due to the thermal relaxation time. This kink goes up with an increase of the thermal relaxation time under LST. The theoretical as well as numerical results are promising for different future applications. 


\section{Acknowledgements}

One of the authors, Dr Nisha Goyal (NG) is thankful to the University Grants Commission, New Delhi for providing financial support through Post Doctoral Fellowship under D S Kothari PDF Scheme bearing No. F.4 - 2/2006(BSR)/MA/15-16/0007.

\section{Nomenclature}

$$
\begin{aligned}
& b, \alpha, \xi, \xi^{*} \text { - voids parameters } \\
& C_{11}, C_{12} \text { - coupling coefficients for CT corresponding to } G \text { and } F \\
& C_{21}, C_{22} \text { - coupling coefficients for GLT corresponding to } G \text { and } F \\
& C_{31}, C_{32} \text { - coupling coefficients for LST corresponding to } G \text { and } F \\
& E_{l}^{*}(l=1,2,3,4) \quad \text { - energy ratios } \\
& e_{i j}-\text { strain tensor } \\
& G, F \text { - coupling parameters } \\
& g \text { - equilibrated body force } \\
& H_{i} \text { - equilibrated stress tensor } \\
& k \text { - wavenumber } \\
& l_{0}, c_{0} \quad \text { - standard length and velocity } \\
& p, \Psi \text { - dilatational potential and shear wave potential } \\
& Q_{i} \quad \text { - heat flux vector and } \\
& t \text { - time } \\
& t_{i j}-\text { stress tensor } \\
& t_{0}, t_{l} \text { - relaxation times } \\
& u_{i}\left(x_{i}, t\right) \text { - components of displacement vector } \boldsymbol{u} \\
& V_{j}, A_{j}(j=1,2,3) \quad \text { - phase speeds and attenuation coefficients } \\
& v_{i}(i=1,2,3) \quad \text { - speed of coupled dilatational waves } P_{I}, P_{I I}, P_{I I I} \\
& v_{4} \text { - speed of shear wave } \\
& x_{i} \text { - spatial coordinate } \\
& \beta, \tau^{*}, m, k_{t}, \zeta, a \quad \text {-thermal parameters } \\
& \delta_{i j}-\text { Kronecker delta } \\
& \eta \text { - entropy per unit mass } \\
& \theta \text { - change in temperature from the constant ambient temperature } T_{0} \\
& \kappa \text { - equilibrated inertia } \\
& \lambda, \mu \text { - Lame's parameters } \\
& \lambda^{*}, \mu^{*}, b^{*}, \alpha^{*}, \gamma^{*} \text { - visco-elastic parameters } \\
& \rho_{0} \quad \text { - density of the medium } \\
& \phi \text { - change in void volume fraction } \\
& \omega \text { - frequency }
\end{aligned}
$$




\section{References}

[1] Iesan D. (2011): On a theory of thermoelastic materials with voids. - J. Elasticity, vol.104, pp.369- 384.

[2] Biot M.A. (1965): Mechanics of Incremental Deformations. - New York.

[3] Szekeres A. (1980): Equation system of thermoelasticity using the modified law of thermal conductivity. - Periodica Polytechnica, Mech. Engng., vol.24, No.3, pp.253-261.

[4] Farkas I. and Szekeres A. (1984): Application of the modified law of heat conduction and state equation to dynamical problems of thermoelasticity. - Periodica Polytechnica, Mech. Engng., vol.28, No.2-3, pp.163-170.

[5] Chandrasekhariah D.S. (1998): Hyperbolic thermoelasticity: A review of recent literature. - Appl. Mech. Rev., vol.51, No.12, pp.705-729.

[6] Szekeres A. and Szalontay M. (1980): Experiments on thermal shock of long rods. - Periodica Polytechnica, Mech. Engng., vol.24, No.3, pp.243-252.

[7] Hetnarski R.B. and Ignaczak J. (1999): Generalized Thermoelasticity. - J. Therm. Stresses, vol.22, pp.451-476.

[8] Lord H.W. and Shulman Y. (1967): A generalized dynamical theory of thermoelasticity. - J. Mech. Phys. Solid., vol.15, pp.299-309.

[9] Green A.E. and Lindsay A. (1972): Thermoelasticity. - J. Elasticity, vol.2, pp.1-7.

[10] Green A.E. and Naghdi P.M. (1993): Thermoelasticity without energy dissipation. - J. Elasticity, vol.31, pp.189-208.

[11] Tzou D.Y. (1995): A unified approach for heat conduction from macro to micro-scales. - J. Heat Trans., vol.117, pp.8-16.

[12] Goodman M.A. and Cowin S.C. (1972): A continuum theory for granular materials. - Arch. Ration. Mech. Anal., vol.44, No.4, pp.249-266.

[13] Nunziato J.W. and Cowin S.C. (1979): A nonlinear theory of elastic materials with voids. - Arch. Ration. Mech. Anal., vol.72, No.2, pp.175-201.

[14] Cowin S.C. and Nunziato J.W. (1983): Linear elastic materials with voids. - J. Elasticity, vol.13, No.2, pp.125147.

[15] Puri P. and Cowin S.C. (1985): Plane waves in linear elastic material with voids. - J. Elasticity, vol.15, No.2, pp.167-183.

[16] Iesan D. (1985): Some theorems in the theory of elastic materials with voids. - J. Elasticity, vol.15, No.2, pp.215-224.

[17] Chandrasekharaiah D.S. (1986): Thermoelasticity with second sound - a review. - Appl. Mech. Rev., vol.39, pp.354-376.

[18] Chandrasekharaiah D.S. (1987): Rayleigh Lamb waves in an elastic plate with voids. - J. Appl. Mech., vol.54, pp.509-512.

[19] Marin M. (1998): Contributions on the uniqueness in thermoelasto-dynamics on bodies with voids. - Cienc. Math. (Havana), vol.16, No.2, pp.101-109.

[20] Birsan M. (2000): Existence and uniqueness of weak solutions in the linear theory of elastic shells with voids. Libertas Mathematica, vol.20, pp.95-105.

[21] Chirita S. and Scalia A. (2001): On the spatial and temporal behaviour in linear thermoelasticity of materials with voids. - J. Therm. Stresses, vol.24, No.5, pp.433-455.

[22] Cicco S.D. and Diaco M. (2002): A theory of thermoelastic materials with voids without energy dissipation. - J. Therm. Stresses, vol.25, No.2, pp.493-503.

[23] Iesan D. and Nappa L. (2004): Thermal stresses in plane strain of porous elastic bodies. - Meccanica, vol.39, pp.125-138. 
[24] Iesan D. (2007): Nonlinear plane strain of elastic materials with voids. - Math. Mech. Solid., vol.11, No.4, pp.361-384.

[25] Tomar S.K. (2005): Wave propagation in a micropolar elastic plate with voids. - J. Vibr. Cont., vol.11, No.6, pp.849-863.

[26] Ciarletta M., Straughan B. and Zampoli V. (2007): Thermo-poroacoustic acceleration waves in elastic materials with voids without energy dissipation. - Int. J. Engng. Sci., vol.45, No.9, pp.736- 743.

[27] Ciarletta M., Svanadze M. and Buonanno L. (2009): Plane waves and vibrations in the theory of micropolar thermoelasticity for materials with voids. - Eur. J. Mech. A/Solids, vol.28, No.4, pp.897-903.

[28] Svanadze M.M. (2014): Potential method in the linear theory of viscoelastic materials with voids. - J. Elasticity, vol.114, pp.101-126.

[29] Chirita S. and Danescu A. (2015): Surface waves in a thermo-viscoelastic porous half-space. - Wave Motion, vol.54, pp.100-114.

[30] Iesan D. (1986): A theory of thermoelastic materials with voids. - Acta Mechanica, vol.60, No.1-2, pp.67- 89.

[31] Dhaliwal R.S. and Wang J. (1993): A heat-flux dependent theory of thermoelasticity with voids. - Acta Mechanica, vol.110, No.1-4, pp.33-39.

[32] Ciarletta M. and Scalia A. (1993): On the nonlinear theory of nonsimple thermoelastic materials with voids. - J. Appl. Math. Mech., vol.73, No.2, pp.67-75.

[33] Ciarletta M. and Scarpetta E. (1995): Some results on thermoelasticity for dielectirc materials with voids. - J. Appl. Math. Mech., vol.75, No.9, pp.707-714.

[34] Tomar S.K., Bhagwan J. and Steeb H. (2014): Time harmonic waves in thermo-viscoelastic material with voids. J. Vibr. Cont., vol.20, pp.1119-1136.

[35] Sharma K. and Kumar P. (2013): Propagation of plane waves and fundamental solution in thermoelastic medium with voids. - J. Therm. Stresses, vol.36, pp.94-111.

[36] Bucur A.V., Passarella F. and Tibullo V. (2014): Rayleigh surface waves in the theory of therm elastic materials with voids. - Mechanica, vol.49, pp.2069-2078.

[37] Bhagwan J. and Tomar S.K. (2016): Reflection and transmission of plane dilatational wave at an interface between an elastic solid and a thermo-viscoelastic solid half-space with voids. - J. Elasticity, vol.121, pp.69-88.

[38] D'Apice C. and Chirita S. (2016): Plane harmonic waves in the theory of thermo-viscoelastic materials with voids. - J. Therm. Stresses, vol.39, pp.142-155.

[39] Santra S., Lahiri A. and Das N.C. (2016): Reflection and refraction of generalized visco-thermoelastic waves at an interface between two half spaces. - Comput. Appl. Math. J., vol.2, No.1, pp.12-22.

[40] Achenbach J.D. (1973): Wave Propagation in Elastic Solids. - North Holland.

[41] Borchardt R.D. (2009): Viscoelastic Waves in Layered Media. - UK: Cambridge University Press.

[42] Mukhopadhyay S. (2000): Effect of thermal relaxation on thermo-viscoelastic interactions in an unbounded body with spherical cavity subjected to periodic loading on the boundary. - J. Therm. Stresses, vol.23, pp.675-684. 